\title{
Genome Editing of the Blood: Opportunities and Challenges
}

\author{
Matthew H. Porteus
}

Published online: 25 January 2015

(C) Springer International Publishing AG 2015

\begin{abstract}
The ability to remove blood cells, including hematopoietic stem cells (HSCs), from a person and then retransplant them [hematopoietic stem cell transplantation (HSCT)] is a well-established treatment paradigm that can be used in both the autologous setting or in the allogeneic setting. Using allogeneic HSCT can cure different genetic diseases of the blood but has significant limitations. An alternative to allogeneic HSCT is to transplant genetically modified HSCs instead. A powerful approach to the precision modification of HSCs is to use genome editing whereby the genome is modified with spatial precision (at an exact location) in the genome and sometimes with nucleotide precision (the exact nucleotide changes are introduced). The progress and challenges of genome editing of blood are discussed.
\end{abstract}

Keywords Genome editing · Homologous recombination · Non-homologous end-joining $\cdot$ Engineered nuclease $\cdot$ Zinc finger nuclease $\cdot$ TAL effector nuclease $\cdot$ CRISPR/Cas9

\section{Introduction}

The modification of the genome of blood cells for therapeutic purposes is a conceptually simple idea that has been the focus of decades of research. In contrast to relatively nonproliferative organs and tissues such as the liver, muscle, and brain, the hematopoietic system is a highly proliferative system. To achieve any sustained therapeutic benefit, therefore, requires that the genome of the blood cells is permanently

This article is part of the Topical Collection on Genome Editing

M. H. Porteus $(\bowtie)$

Department of Pediatrics MC5462, Stanford University, Stanford, CA 94305, USA

e-mail: mporteus@stanford.edu modified rather than using a non-integrating, non-replicating vector that would be quickly diluted. The primary approach to genome modification of blood cells has been to use integrating viral vectors, primarily retroviral and lentiviral vectors. Using these vectors, a transgene that is driven by an artificial promoter is integrated into the genome in an uncontrolled fashion. Important work has demonstrated that retroviral and lentiviral vectors do not integrate in a random fashion but instead have biased integration pattern [1]. Retroviral vectors, for example, are biased towards integrating into the promoter regions of actively expressed genes, while lentiviral vectors are biased to integration more broadly into the $5^{\prime}$ region of actively expressed genes. This bias, while not absolute, has effects on the relative safety of the two vectors. All of the clinical trials using retroviral vectors except for one have shown that the first generation of $\gamma$-retroviral vectors in which the LTR is still active, can cause leukemia by the activation of proto-oncogenes by the viral integration [2-5]. In some cases, all or almost all of the patients developed a serious adverse event of this nature $[2,3]$. While the number of patients treated with lentiviral vectors is smaller and the follow-up is shorter, there has been no report of a leukemia caused by insertional activation of an oncogene from a lentiviral vector $[6,7 \cdot, 8]$. Nonetheless, there remain concerns about the long-term safety of even these vectors because in mice that are sensitized to the development of lymphoma, even the "safest" lentiviral constructs still decreased the time to development of cancer as compared to controls (decreased latency) [9]. While all lentiviral constructs led to a decreased latency, the types of genes that led to the decreased latency changed with the different constructs. In some versions, the decrease in latency was the result of oncogene activation, while in the other constructs, the decrease in latency was the result of disrupting tumor suppressor genes.

An alternative approach to using gene therapy with integrating viral vectors is to use genome editing to precisely and 
permanently modify the genome of cells. Genome editing generally encompasses the approach of modifying the genome in a highly controlled fashion. It can be done using engineered nucleases or by the homologous integration of AAV vectors. Through mechanisms that are still not entirely understood, when an AAV vector is delivered into cells in which a transgene is flanked by regions of homology to a target locus in the genome, the AAV vector will integrate precisely into the genome guided by the regions of homology [10]. This homologous integration event can occur in up to $1 \%$ of cells and, if designed properly, can form the clinical basis of a potentially highly effective therapeutic $[11 \bullet, 12]$. This review will focus on genome editing using engineered nucleases for genome editing (Fig. 1).

Genome editing using engineered nucleases is fundamentally based on designing an enzyme (in this case, a nuclease) that can make a DNA double-strand break (DSB) at a specific location in the genome. This DSB activates the cell's endogenous break repair machinery. There are two basic ways that cells repair DSB. The first is by non-homologous end-joining (NHEJ) in which the two ends are simply ligated back together. In the case of a break that has clean ends as is often created by an engineered nuclease, the NHEJ machinery will usually repair the break in a precise fashion, leaving the genome unchanged. Occasionally, however, the NHEJ mechanism creates a small insertion or deletion (in/del) at the site of the break, perhaps because the break is no longer clean because of action of an exonuclease, thereby creating a small mutation in the genome at a spatially precise location. The second mechanism that cells use to repair double-strand breaks is by homologous recombination (HR). In HR, the cell uses a "copy and paste" mechanism whereby it finds a piece of DNA that has homology to the region flanking the break and uses that piece of homologous DNA as a template to synthesize new DNA and then recombine the newly made DNA into the area of the break. The usual DNA template for HR is the sister chromatid, an identical copy of the damaged chromosome. But for reasons that are not yet understood, the HR machinery can also use exogenously supplied DNA (a "donor vector") as the template for repair. By designing the donor vector to have regions of homology (at least 400 base pairs) that flank genetic changes, precise nucleotide changes can be introduced at the site of break [13-15]. These changes can be as small as a single base pair or as large as a cassette of genes encompassing $>10$ kilobases of DNA $[16 \cdot, 17]$. In this way, HR-mediated genome editing permanently modifies the genome with precise spatial AND nucleotide resolution.

There are now multiple different engineered nuclease platforms that can be used for genome editing including meganucleases, zinc finger nucleases (ZFNs), TAL effector nucleases (TALENs), RNA-guided endonucleases (CRISPR/ Cas9), and hybrid platforms such mega-TALs and CRISPRFn nucleases. ZFNs and TALENs share a similar structure in which a DNA binding domain is fused to the non-specific nuclease domain from the type IIS FokI restriction endonuclease. The specificity of ZFNs and TALENS is derived from engineering the DNA binding domain to recognize a specific target sequence. For ZFNs, this domain is engineered by assembling individual zinc finger domains (each of which mediates binding to 3-4 nucleotides) to create an array containing 3-6 zinc fingers domains (thus binding a target sequence of 9-18 base pairs). For TALENs, the DNA binding domain is engineered by stringing together individual 34 amino TAL effector repeats [each repeat mediates binding to a single base pair through a 2-amino-acid repeat divariable residues (RVD)]. In order to achieve sufficient binding energy to mediate specific binding, an array of 12-20 TAL effector repeats needs to be generated. For both ZFNs and TALENs, since the nuclease domain needs to form a dimer to be active, a pair of nucleases needs to be engineered in order to efficiently cut a specific site in the genome. These proteins are engineered nucleases that do not exist in nature. In contrast to the
Fig. 1 Schematic of different uses of genome editing to modify the behavior of blood cells

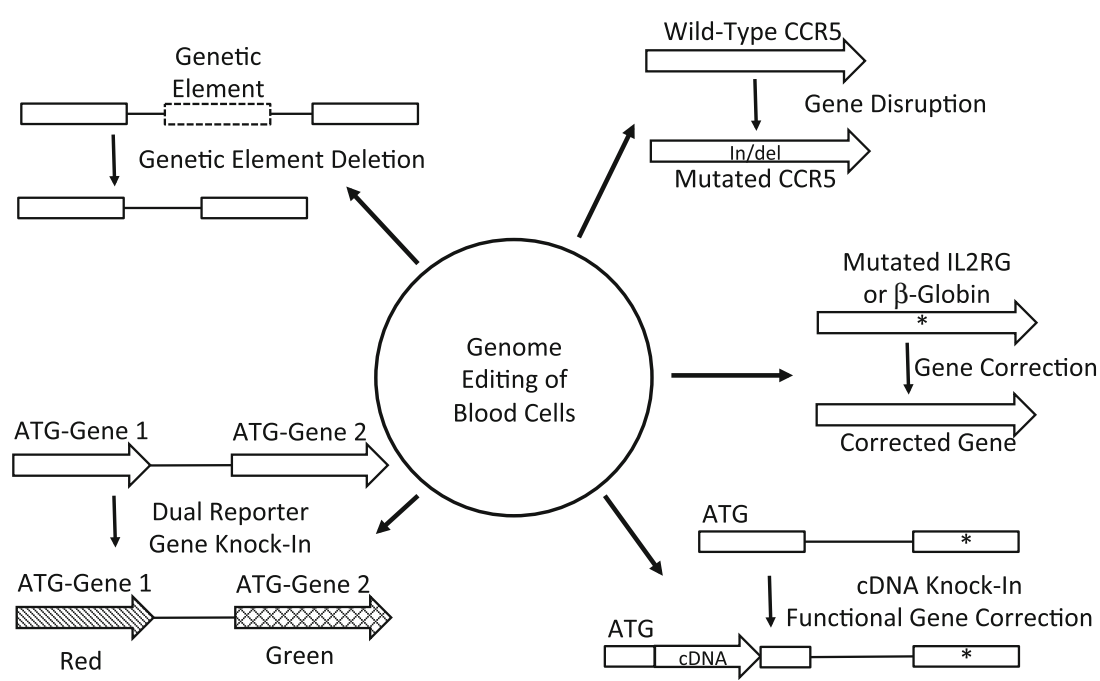


protein-DNA interaction that mediates ZFN and TALEN specificity, specificity of CRISPR/Cas9 is mediated by RNA-DNA hybridization. In the CRISPR/Cas9 system, a guide RNA is made in which the target 20 base pair guide sequence is designed to hybridize with a specific genomic site. This guide RNA in conjunction with a linker RNA (called a TRACR RNA) complexes with the Cas9 protein, and this guide RNA/Cas9 protein complex is able to scan the entire genome to find the appropriate target sequence, unwind the double-strand DNA to allow pairing of the RNA guide sequence with the genomic target, and then create a break at that sequence. This system evolved to mediate resistance to invading DNA molecules in bacteria, and it is quite amazing that it is so readily adopted and functional in editing the genomes of mammalian cells. All three platforms have been shown to be active in a wide variety of cell types and organisms. They all fundamentally create a DSB in the genome, but the type of DSB is different between the three platforms with ZFNs creating breaks that leave clean $5^{\prime}$ overhangs, TALENs leaving breaks that leave clean $5^{\prime}$ overhangs but also can create more complicated overhangs, and the CRISPR/Cas9 nuclease leaving blunt breaks. The different types of breaks may have subtle differences on genome editing outcomes at different loci [13].

Below, I discuss three different blood disease targets for which genome editing has been applied: severe combined immunodeficiency caused by mutations in the IL2RG gene (SCID-X1), HIV, and the $\beta$-hemoglobinopathies.

\section{Genome Editing for SCID-X1}

SCID-X1 is a devastating genetic disease caused by mutations throughout the IL2RG gene leading to a lack of T cells. This disease can be cured by allogeneic bone marrow transplantation, which highlights that replacing the lymphoid or hematopoietic system with one that does not contain a mutation in the IL2RG gene can be curative. The goal of genome editing is to replace the hematopoietic system, or at least the lymphoid system, with autologous cells that no longer have a dysfunctional IL2RG gene instead of having to use allogeneic cells.

A series of ZFNs were made to target exon 5 of the IL2RG, a hotspot for mutations causing SCID-X1 [17]. This series was screened using a GFP-based reporter assay to identify the most active pairs although now that screening process would be most efficiently performed by determining the frequency of in/dels in the endogenous gene in a cell line. Nonetheless, the screen identified a lead pair that was most active. The investigators then created a series of variants of both the left and right ZFN to further optimize their on-target editing activity. From this evaluation came a single pair that had the best activity in stimulating gene correction in a reporter gene. Interestingly, this optimized pair also showed loss toxicity than prior pairs of ZFNs that had been described. Using these ZFNs, a gene editing frequency of $18 \%$ was achieved in human erythroleukemia cell line (K562 cells) and of $4 \%$ in primary T cells [17]. These results were the first demonstration that genome editing by homologous recombination using designer nucleases could reach frequencies in cell types that were therapeutically relevant.

Since the initial report of these ZFNs they have continued to be developed for potential clinical use. The major target cell type in these subsequent studies has been in human CD $34^{+}$ hematopoietic stem and progenitor cells (HSPCs). When both the donor and $\mathrm{ZFNs}$ were delivered into $\mathrm{CD} 34^{+}$cells as integration defective lentivirus (IDLV), the rate of targeted genome editing by homologous recombination was $0.1 \%$ [18]. This was an extremely encouraging proof of concept that CD34 ${ }^{+}$HSPCs are amenable to genome editing by homologous recombination using engineered nucleases. Experiments of nature have shown that even a single corrected precursor can be sufficient to reconstitute a robust $\mathrm{T}$ cell immune system, and thus, even this low frequency of editing might have been clinically relevant [19].

Subsequently, the Naldini group has continued to refine the process of genome editing in $\mathrm{CD} 34^{+}$cells at exon 5 of the IL2RG gene using these ZFNs [20••]. These refinements have included delivering the ZFNs as messenger RNAs (mRNAs) via electroporation rather than as IDLV, timing the delivery to the donor through IDLV and the mRNA ZFNs appropriately, and optimizing the culture conditions of the $\mathrm{CD} 34^{+}$cells. With these refinements, there was a $>10$-fold increase in the frequency of genome editing by homologous recombination to an overall frequency of $2-10 \%$. Moreover, the edited cells were capable of multi-lineage reconstitution of the hematopoietic system following transplantation into immunodeficient mice and to be able to generate functional $\mathrm{T}$ cells that proliferated and responded to cytokine stimuli as would unmodified, wild-type $T$ cells would.

As discussed below, there are some potential advantages of TALENs and CRISPRs in regards to off-target effects and even on-target efficiency that make them excellent candidates for further development to current mutations in SCID-X1. These results in $\mathrm{CD} 34^{+}$cells with ZFNs are the farthest advanced in the clinical development of a gene correction-based genome editing strategy.

\section{Genome Editing to Generate HIV Resistance}

The human immunodeficiency virus (HIV) now infects $\sim 34$ million people worldwide and is the causative agent for the acquired immunodeficiency syndrome (AIDS). Development of highly active anti-retroviral therapy (HAART) has transformed HIV infection in the developed world from one that would lead to the inevitable destruction of the immune system 
and subsequent death from opportunistic infections, to a chronic disease. There are limitations to HAART, however, including the need for daily treatment for the rest of the patient's lifetime, its relative unavailability in the developing world, and side effects that lead some patients to discontinue therapy and others to suffer significant iatrogenic morbidity. Just as the close study of a human patient who had a spontaneous single reversion in a precursor cell ("a human experiment of nature") highlighted the need that only a small number of HSPCs might need to be corrected to cure SCID-X1, the close study of the genetics of people who are resistant to infection by HIV points to a potential genome editing strategy that might use genome editing and genetics to replace the need to take anti-retroviral molecules every day for the rest of one's life.

HIV can be distinguished into two broad sub-categories based on whether it uses the CCR5 or CXCR4 protein as a co-receptor for viral entry into the cell (CCR5-tropic or R5HIV vs CXCR4-tropic or X4-HIV). There is a small subpopulation of people who have a 32-base-pair deletion $(\Delta 32)$ in the CCR5 coding region that causes a null mutation in the CCR5 gene. When people are homozygous for the $\Delta 32$ allele, they are resistant to HIV infection [21]. The mechanism of resistance is that, while the X4 tropic strain of HIV can develop once a patient is infected and is a predictor of more aggressive disease, the $\mathrm{X} 4$ strain does not transmit between people and only the R5 strain is transmitted between people. People who are homozygous deficient in CCR5, therefore, have no receptor for HIV to initiate cellular entry upon infection, and thus, they are resistant to HIV. This suggests that a genome editing strategy of creating spatially specific mutations in the CCR5 gene might be a way of generating HIVresistant immune cells that would be resistant to destruction by the virus.

The concept of generating an HIV-resistant immune system is also supported by the case of the "Berlin" patient [22]. This HIV-infected individual developed AML, a lethal form of leukemia, for which the best therapy can be an allogeneic hematopoietic stem cell transplant (allo-HSCT). The patient and his doctor decided to search the donor database for a donor who was homozygous for the $\Delta 32$ CCR5 allele and found one. They then performed an allo-HSCT with the stem cells from this donor, and following transplantation, the patient has had no evidence of HIV as determined by some of the most sophisticated laboratories in the world. While there may have been multiple other reasons that contributed to the clearance of the virus [the lack of a functionally demonstrated X4-tropic virus prior to transplant, a low HIV viral load prior to transplant, the use of anti-T cell chemotherapy to eliminate latently infected $\mathrm{T}$ cells, cytotoxic chemotherapy which may have eliminated all HIV-infected blood cells, graft vs host disease (GVHD) that may have further eliminated any residual infected cells, and the use of anti-GVHD medicines that may have further eliminated host viral susceptible cells], there is strong evidence that the donor cells that were homozygous $\Delta 32$ and thereby resistant to infection were an essential factor. Thus, instead of using allogeneic cells that are CCR5 mutant, the logic is to use genome editing of autologous cells to make them HIV resistant by inducing mutations in the CCR5 gene.

\section{Mutating CCR5 in T Cells Using Engineered Nucleases}

ZFNs, TALENs, and CRISPR/Cas9 nucleases have all been engineered to target the coding sequence of the CCR5 gene [23-27]. When the different platforms have been compared, the activity of each nuclease has been relatively equivalent in causing in/dels in the CCR5 gene. The clinical development of ZFNs targeting CCR5 is by far the most advanced and will be the primary focus here $[28 \cdot 0]$. While not explicitly reported, the design of the ZFNs targeting CCR5 was similar to the design of ZFNS targeting exon 5 of the IL2RG gene in that multiple candidate ZFNs were screened, the most promising identified and then further variants of those were generated to identify the best pair [23]. The specific details of that lengthy, sophisticated, and expensive process are proprietary to Sangamo Biosciences. The ultimate pair identified for further development targets the first transmembrane domain of the seven-transmembrane CCR5 protein and lies $5^{\prime}$ to the $\Delta 32$ mutation that is found in human patients. When the CCR5 ZFNs were expressed in cells, they induced breaks in the CCR5 gene, which were then repaired in a mutagenic fashion by NHEJ. Mutations at the CCR5 ZFN binding site created null mutations as demonstrated by the lack of expression of CCR5 on the cell surface. The ability to create HIV-resistant T cells was then tested by introducing the CCR5 ZFNs via an adenoviral vector in ex vivo expanded primary $\mathrm{T}$ cells. The frequency of allelic mutation in primary T cells was initially in the range of $2-15 \%$, but after HIV challenge, either in vitro or in vivo after transplantation of the modified $\mathrm{T}$ cells into immunodeficient mice, the frequency of modified cells increased substantially to $30-55 \%$ (in vitro challenge) or 6-44 \% (in vivo challenge). These mouse studies were the foundation efficacy studies leading to a phase I clinical trial.

In the phase I clinical trial, primary $\mathrm{T}$ cells from HIVinfected patients were harvested, expanded, and transduced with the CCR5 ZFNs via an adenoviral vector [28••]. The overall rate of allele modification was $11-28 \%$ prior to infusion of the autologous cell product. After infusion, the investigators demonstrated that the modified cells persisted over time and seemed to home to extravascular sites appropriately. The phase I trial was deemed a success because the process was feasible and no serious adverse events occurred. The evidence of efficacy was mixed as there were subtle but not consistent changes in viral loads and CD4 T cell counts among the patients. Even during treatment interruptions in which 
HAART therapy was discontinued, there was no clear demonstration that CCR5-modified cells were enriched, although the authors claim that the modified cells had a slightly longer half-life than unmodified cells. The trial is now being expanded into a phase II trial.

\section{Mutating CCR5 in CD34 ${ }^{+}$Hematopoietic Stem and Progenitor Cells}

While editing mature $\mathrm{T}$ cells has the potential advantage of being safer as there is a decreased likelihood that this terminally differentiated cell will transform, an important potential limitation is that it may be impossible to edit a sufficient number of mature $\mathrm{T}$ cells to create the diverse $\mathrm{T}$ cell repertoire needed to protect a patients from AIDS defining infections, and thus, it would not be sufficient to stop HAART permanently. The potential solution to this problem is to use engineered nucleases to inactivate the CCR5 gene in CD34 ${ }^{+}$ HSPCs. Moreover, HIV is also able to infect cell types that are not $\mathrm{T}$ cells, including macrophages, and the strategy of creating resistant $\mathrm{T}$ cells would not create a generally HIV-resistant immune system.

The same ZFNs that were used to mutate the CCR5 gene in primary $\mathrm{T}$ cells have also been used to mutate the CCR5 gene in human CD34 $4^{+}$HSPCs [29]. In these studies, the CCR ZFNs were delivered by electroporation into $\mathrm{CD} 34^{+}$cells as DNA expression plasmids, and an allele modification frequency of $\sim 16 \%$ was obtained. As techniques have advanced, nucleases are now being delivered as mRNAs via electroporation to cells. These modified cells could give rise to multi-lineage hematopoiesis following transplantation into immunodeficient (NSG) mice. In the mouse model, human T cells with mutations in the CCR5 gene developed, and after HIV challenge, the allele modification frequency of the CCR5 gene increased from 5 to $10 \%$ prior to challenge to $\sim 40$ to $80 \%$ after HIV challenge. Moreover, the CD4/CD8 ratio was preserved after HIV challenge. All of these results support the idea of the potential efficacy of mutating the CCR5 gene in CD34 $4^{+}$HSPCs using engineered nucleases as a method to create an HIV-resistant immune system.

As discussed above, engineered nucleases from all of the major platforms have been designed to target CCR5. In most of these studies, a single break was created, and mutations in CCR5 were generated by the random insertions/deletions created by mutagenic NHEJ. In Mandal et al. [27], they developed an interesting variant of this strategy using the CRISPR/ Cas9 system. In this system, they used two guide RNAs to target two sites in the CCR5 coding region. In doing so, they were utilizing the prior observation that, by making simultaneous breaks on the same chromosome, one can create defined deletions [30]. By creating two simultaneous breaks and enriching for transfected CD $34^{+}$HSPCs, they were able to increase the allele modification frequency from $\sim 14 \%$ with a single guide to $\sim 42 \%$ using the dual guide approach with $\sim 19-26 \%$ of the cells having a biallelic disruption of the CCR5 gene. Importantly, using the dual nuclease/guide approach, the deletion that disrupts the CCR5 gene is more highly defined often with a precise nucleotide junction than when a single nuclease is used.

\section{Creating Multi-layered Genetic Resistance to HIV}

One of the hallmarks of HIV is its ability to mutate and evolve. In this way, it can change its ability to enter cells through the CXCR4 co-receptor instead of CCR5, and it can develop resistance to small molecule inhibitors of its viral lifecycle. Thus, potential drawbacks of a CCR5 knockout-only approach is that it would not be effective in patients who have already be infected with X4-tropic HIV and may not be sufficient to prevent the large number of HIV virions already present in an infected patient from mutating, evolving, and escaping to find another mechanism of entry. The concept would be to use genome editing to create multiple genetic barriers to the HIV lifecycle just as HAART small molecule therapy blocks the HIV lifecycle at multiple steps. As a proof-of-concept in a human T cell HIV reporter line, Voit et al. [31] used genome editing by homologous recombination to knock-in various anti-HIV genes into the CCR5 locus. In this way, the CCR5 gene is still mutated, but additional genetic roadblocks are added. One of the key additional roadblocks was derived from an understanding of HIV restriction in non-human primates. Rhesus macaques are resistant to HIV infection because they express the rhesus form of TRIM $5 \alpha$. In contrast to the human TRIM $5 \alpha$, the rhesus version can target the invading HIV virion for degradation by the proteasome after entry but prior to trafficking to the nucleus. These investigators demonstrated that, by stacking genetic elements to confer HIV resistance, there was a quantitative increase in cellular resistance to both R5- and X4-tropic HIV. While these are intriguing proof-ofconcept studies, they have not been validated using this strategy to modify primary human T cells and human HSPCs and challenging those cells both ex vivo and in vivo with R5- and X4-tropic HIV.

\section{Genome Editing for the Hemoglobinopathies}

Another important class of diseases that may be amenable to treatment using genome editing is the hemoglobinopathies, which can be sub-categorized into sickle cell disease, $\beta$-thalassemia, and $\alpha$-thalassemia. There have been a number of interesting studies using genome editing as a potential therapeutic approach to these diseases. These studies can be 
divided into editing approaches that edit induced pluripotent stem cells vs editing approaches that focus on somatic cells.

\section{Genome Editing of Pluripotent Cells}

The underlying principle of editing pluripotent cells to cure hemoglobinopathies was demonstrated by Hanna et al. [32]. They reprogrammed skin cells from a mouse model of sickle cell disease into induced pluripotent cells (iPSCs) [32]. They then used non-nuclease mediated homologous recombination, a process that is several logs less efficient than nucleaseinduced homologous recombination, to correct the sickle cell mutation in the iPS cells. They then differentiated the genetically corrected iPS cells into hematopoietic progenitor (HP) cells by directed differentiation using HoxB4, a known leukemia-associated gene in humans. HP cells derived from the genetically corrected iPS cells were then transplanted back into a syngeneic mouse (equivalent to an autologous transplant) where they generated non-sickling red blood cells. Thus, while many inefficiencies exist in this process, most notably the differentiation of pluripotent cells into true hematopoietic stem cells, this was a seminal proof-of-concept piece of work validating a process in which genetically corrected patient derived iPS cells might be used to cure genetic blood diseases.

While the gene correction by homologous recombination in mouse iPS cells is low, it is even more difficult in human iPS cells. In fact, despite much excitement, the ability to edit human iPS cells without using engineered nucleases or AAV has been too difficult to be broadly adopted. There have been two groups, however, that have shown that ZFNs can be used to stimulate gene correction in iPS cells derived from sickle cell patients at the HBB locus itself [33, 34]. The overall efficiency was still very low, but because iPS clones can be expanded nearly indefinitely, the low efficiency is somewhat mitigated.

An alternative use of genome editing to directly correct a disease causing mutation is to use a "safe harbor" approach whereby a wild-type copy of the mutant gene of interest is integrated into a precise location in the genome using nuclease-mediated genome editing in iPS cells. This approach has been used in proof-of-concept studies in human iPS cells derived from patients with $\alpha$-thalassemia and with chronic granulomatous disease, a genetic disease causing defects in neutrophil function $[35,36]$.

\section{Genome Editing of Somatic Cells}

There have been three major applications of genome editing of somatic cells that are directly applicable to genetic diseases of the blood: (1) the development of a functional gene correction, complementary DNA (cDNA) knock-in approach; (2) the creation of dual globin endogenous globin gene reporters lines for drug screening; and (3) the validation of a regulatory genetic element as a target for de-repression of the $\gamma$-globin gene.

While sickle cell disease is caused by a single point mutation in the $\beta$-globin gene, $\beta$-thalassemia is caused by mutations throughout the $\beta$-globin gene. While in the future genome editing may be become sufficiently streamlined to allow patient specific gene correction for $\beta$-thalassemia, currently, the field is not so advanced. Voit et al. [16•] described a system that would functionally correct almost all of the mutations that cause $\beta$-thalassemia through a cDNA knock-in approach. For this approach, they designed highly active nucleases to target the region adjacent to the initiation ATG of exon 1 of the $\beta$ globin gene. They used a donor construct in which a wild-type copy of the $\beta$-globin cDNA would be knocked into the endogenous locus such that the cDNA would be driven by the endogenous regulatory elements and endogenous ATG start codon. Moreover, the knock-in cassette also contained a chemotherapy selection gene (P140K MGMT) to subsequently enrich for functionally corrected cells. Using this system in K562 cells, a human erythroleukemia cell line, they were able to functionally correct $>20 \%$ of cells initially and, after a single round of chemoselection, were able to increase the percentage of corrected cells to $\sim 100 \%$. While this strategy preserves the regulatory elements in the endogenous gene, including downstream intronic elements, the cDNA knock-in strategy would cause any regulation through splicing or alternative splicing to be lost. Preliminary experiments, however, demonstrate that the functionally corrected allele expresses equivalent transcripts to the wild-type allele in erythroid cells derived from $\mathrm{CD} 34^{+}$cells in culture, and thus, at least at this locus, the issue of splicing-based regulation of a cDNA expressed from the endogenous locus may not be significant.

Voit et al. [16•] also used genome editing to create a dualglobin reporter system as proof-of-concept to validate that such cell lines would be useful in screening for drugs that would preferentially upregulate $\gamma$-globin as compared to $\beta$ globin. In these experiments, they knocked enhanced green fluorescent protein (EGFP) into $\beta$-globin locus such that EGFP would be expressed from the endogenous $\beta$-globin ATG and knocked tdTomato into the $\gamma$-globin locus such that tdTomato would be expressed from the endogenous $\gamma$-globin ATG. In this way, a drug that preferentially turned the cells more red than green would identify a candidate molecule that would preferentially upregulate $\gamma$-globin. They found a number of compounds that preferentially upregulated $\gamma$-globin including hydroxyurea, the only FDA-approved drug for this purpose. The preferential upregulation of $\gamma$-globin is particularly important in sickle cell disease where an increase in $\gamma$-globin can disrupt the kinetics of sickling in red blood cells in sickle cell diseases without causing a concomitant increase in the expression of the pathologic sickle $\beta$-globin. 
Lee et al. [30] was the first to demonstrate that defined deletions could be generated by the simultaneous creation of two DSBs by a pair of engineered nucleases. That is, by engineering nucleases to cut two sites on the same chromosome, one can generate defined deletions between the two breaks. Bauer et al. [37••] had used sophisticated genomics data and experimentation to identify a putative lineage specific regulatory element for the Bcl11a gene. Specifically, they had identified a 10kilobase region that they thought contained a specific erythroid enhancer that turned Bcll1a on in erythroid cells. To validate the activity of this enhancer they used engineered nucleases to specifically delete the enhancer in erythroid cells and B cells and showed that cells with biallelic deletion of the element in erythroid cells abrogated Bcl11a expression in those cells but did not perturb expression in B cells [37••]. Thus, the genome editing definitively and functionally validated the genomics data. Moreover, the biallelic deletion of the enhancer in erythroid cells also resulted in the $>100$-fold upregulation of $\gamma$-globin, thus also validating the enhancer as a potential target for genome editing therapeutic approach. That is, instead of using homologous recombination to try to correct the mutations causing $\beta$-hemoglobinopathies, one could use engineered nucleases to delete a specific regulatory element that resulted in the de-repression of $\gamma$ globin genetically, instead of having to use a small molecule like hydroxyurea to achieve the same purpose.

\section{Conclusions}

In summary, the last decade has seen tremendous advancements in the use of genome editing to both better understand the mechanisms of genetic diseases of the blood and as a therapeutic to precisely modify blood cells. This advance has been the result of the development of a more robust set of tools in the tool kit, particularly with the development of the TALEN and CRISPR/Cas9 nuclease platforms, and a more sophisticated way of thinking about how to use those tools. There are strong reasons to believe that, in the next decade, the lives of patients will be transformed by the use of genome editing of blood cells.

\section{Compliance with Ethics Guidelines}

Conflict of Interest Matthew H. Porteus reports personal fees from CRISPR Therapeutics.

Human and Animal Rights and Informed Consent This article does not contain any studies with human or animal subjects performed by any of the authors.

\section{References}

Papers of particular interest, published recently, have been highlighted as:

- Of importance

• Of major importance

1. Bushman F, Lewinski M, Ciuffi A, Barr S, Leipzig J, Hannenhalli $\mathrm{S}$, et al. Genome-wide analysis of retroviral DNA integration. Nat Rev Microbiol. 2005;3(11):848-58. doi:10.1038/nrmicro1263.

2. Braun CJ, Boztug K, Paruzynski A, Witzel M, Schwarzer A, Rothe $\mathrm{M}$, et al. Gene therapy for Wiskott-Aldrich syndrome-long-term efficacy and genotoxicity. Sci Transl Med. 2014;6(227):227ra33. doi:10.1126/scitranslmed.3007280.

3. Ott MG, Schmidt M, Schwarzwaelder K, Stein S, Siler U, Koehl U, et al. Correction of X-linked chronic granulomatous disease by gene therapy, augmented by insertional activation of MDS1EVI1, PRDM16 or SETBP1. Nat Med. 2006;12(4):401-9.

4. Hacein-Bey-Abina S, Hauer J, Lim A, Picard C, Wang GP, Berry $\mathrm{CC}$, et al. Efficacy of gene therapy for X-linked severe combined immunodeficiency. N Engl J Med. 2010;363(4):355-64. doi:10. 1056/NEJMoa1000164.

5. Aiuti A, Cattaneo F, Galimberti S, Benninghoff U, Cassani B, Callegaro L, et al. Gene therapy for immunodeficiency due to adenosine deaminase deficiency. N Engl J Med. 2009;360(5): 447-58. doi:10.1056/NEJMoa0805817.

6. Hacein-Bey-Abina S, Pai SY, Gaspar HB, Armant M, Berry CC, Blanche $\mathrm{S}$, et al. A modified gamma-retrovirus vector for X-linked severe combined immunodeficiency. N Engl J Med. 2014;371(15): 1407-17. doi:10.1056/NEJMoa1404588.

7. Aiuti A, Biasco L, Scaramuzza S, Ferrua F, Cicalese MP, Baricordi $\mathrm{C}$, et al. Lentiviral hematopoietic stem cell gene therapy in patients with Wiskott-Aldrich syndrome. Science. 2013;341(6148): 1233151. doi:10.1126/science.1233151. The first use of a lentiviral vector to genetically modify hematopoietic stem cells to treat a genetic disease of the blood.

8. Biffi A, Montini E, Lorioli L, Cesani M, Fumagalli F, Plati T, et al. Lentiviral hematopoietic stem cell gene therapy benefits metachromatic leukodystrophy. Science. 2013;341(6148):1233158. doi:10. 1126/science. 1233158.

9. Cesana D, Ranzani M, Volpin M, Bartholomae C, Duros C, Artus $\mathrm{A}$, et al. Uncovering and dissecting the genotoxicity of selfinactivating lentiviral vectors in vivo. Mol Ther J Am Soc Gene Ther. 2014;22(4):774-85. doi:10.1038/mt.2014.3.

10. Russell DW, Hirata RK. Human gene targeting by viral vectors. Nat Genet. 1998;18(4):325-30.

11. Barzel A, Paulk NK, Shi Y, Huang Y, Chu K, Zhang F, et al. Promoterless gene targeting without nucleases ameliorates haemophilia B in mice. Nature. 2014. doi:10.1038/nature13864. The use of AAV mediated genome editing without a nuclease to correct a mouse model of a human disease.

12. Melo SP, Lisowski L, Bashkirova E, Zhen HH, Chu K, Keene DR, et al. Somatic correction of junctional epidermolysis bullosa by a highly recombinogenic AAV variant. Mol Ther J Am Soc Gene Ther. 2014;22(4):725-33. doi:10.1038/mt.2013.290.

13. Hendel A, Kildebeck EJ, Fine EJ, Clark JT, Punjya N, Sebastiano $\mathrm{V}$, et al. Quantifying genome-editing outcomes at endogenous loci with SMRT sequencing. Cell Rep. 2014;7(1):293-305. doi:10. 1016/j.celrep.2014.02.040.

14. Porteus MH, Baltimore D. Chimeric nucleases stimulate gene targeting in human cells. Science. 2003;300(5620):763.

15. Porteus MH. Mammalian gene targeting with designed zinc finger nucleases. Mol Ther J Am Soc Gene Ther. 2006;13(2):438-46. 
16. Voit RA, Hendel A, Pruett-Miller SM, Porteus MH. Nucleasemediated gene editing by homologous recombination of the human globin locus. Nucleic Acids Res. 2014;42(2):1365-78. doi:10. 1093/nar/gkt947. The first use of "functional" gene correction whereby a wild-type cDNA is inserted by genome editing precisely into its own locus.

17. Urnov FD, Miller JC, Lee YL, Beausejour CM, Rock JM, Augustus S, et al. Highly efficient endogenous human gene correction using designed zinc-finger nucleases. Nature. 2005;435(7042):646-51.

18. Lombardo A, Genovese P, Beausejour CM, Colleoni S, Lee YL, Kim KA, et al. Gene editing in human stem cells using zinc finger nucleases and integrase-defective lentiviral vector delivery. Nat Biotechnol. 2007;25(11):1298-306. doi:10.1038/nbt1353.

19. Bousso P, Wahn V, Douagi I, Horneff G, Pannetier C, Le Deist F, et al. Diversity, functionality, and stability of the T cell repertoire derived in vivo from a single human $\mathrm{T}$ cell precursor. Proc Natl Acad Sci U S A. 2000;97(1):274-8.

20.• Genovese P, Schiroli G, Escobar G, Di Tomaso T, Firrito C, Calabria A, et al. Targeted genome editing in human repopulating haematopoietic stem cells. Nature. 2014;510(7504):235-40. doi: 10.1038/nature13420. The first use of nuclease mediated functional gene correction of a disease associated gene in hematopoietic stem cells at frequencies that would be therapeutically relevant.

21. Zimmerman PA, Buckler-White A, Alkhatib G, Spalding T, Kubofcik J, Combadiere C, et al. Inherited resistance to HIV-1 conferred by an inactivating mutation in $\mathrm{CC}$ chemokine receptor 5: studies in populations with contrasting clinical phenotypes, defined racial background, and quantified risk. Mol Med. 1997;3(1): 23-36.

22. Hutter G, Nowak D, Mossner M, Ganepola S, Mussig A, Allers K, et al. Long-term control of HIV by CCR5 Delta32/Delta32 stemcell transplantation. N Engl J Med. 2009;360(7):692-8. doi:10. 1056/NEJMoa0802905.

23. Perez EE, Wang J, Miller JC, Jouvenot Y, Kim KA, Liu O, et al. Establishment of HIV-1 resistance in CD4+ T cells by genome editing using zinc-finger nucleases. Nat Biotechnol. 2008;26(7): 808-16. doi:10.1038/nbt1410.

24. Miller JC, Tan S, Qiao G, Barlow KA, Wang J, Xia DF, et al. A TALE nuclease architecture for efficient genome editing. Nat Biotechnol. 2011;29(2):143-8. doi:10.1038/nbt.1755.

25. Mussolino C, Morbitzer R, Lutge F, Dannemann N, Lahaye T, Cathomen T. A novel TALE nuclease scaffold enables high genome editing activity in combination with low toxicity. Nucleic Acids Res. 2011;39(21):9283-93. doi:10.1093/nar/gkr597.

26. Lin Y, Cradick TJ, Brown MT, Deshmukh H, Ranjan P, Sarode N, et al. CRISPR/Cas9 systems have off-target activity with insertions or deletions between target DNA and guide RNA sequences.
Nucleic Acids Res. 2014;42(11):7473-85. doi:10.1093/nar/ gku402.

27. Mandal P, Ferreira L, Collins R, Meissner T, Boutwell C, Friesen $\mathrm{M}$, et al. Efficent ablation of genes in human hematopoietic stem and effector cells using CRISPR/Cas9. Cell Stem Cell. 2014;15: 643-52. doi:10.1016/j.stem.2014.10.004.

28.• Tebas P, Stein D, Tang WW, Frank I, Wang SQ, Lee G, et al. Gene editing of CCR5 in autologous CD4 T cells of persons infected with HIV. N Engl J Med. 2014;370(10):901-10. doi:10.1056/ NEJMoa1300662. The first clinical trial in which nuclease modified blood cells were given to patients.

29. Holt N, Wang J, Kim K, Friedman G, Wang X, Taupin V, et al. Human hematopoietic stem/progenitor cells modified by zincfinger nucleases targeted to CCR5 control HIV-1 in vivo. Nat Biotechnol. 2010;28(8):839-47. doi:10.1038/nbt.1663.

30. Lee HJ, Kim E, Kim JS. Targeted chromosomal deletions in human cells using zinc finger nucleases. Genome Res. 2010;20(1):81-9. doi:10.1101/gr.099747.109.

31. Voit RA, McMahon MA, Sawyer SL, Porteus MH. Generation of an HIV resistant T-cell line by targeted "stacking" of restriction factors. Mol Ther J Am Soc Gene Ther. 2013;21(4):786-95. doi: 10.1038/mt.2012.284.

32. Hanna J, Wernig M, Markoulaki S, Sun CW, Meissner A, Cassady JP, et al. Treatment of sickle cell anemia mouse model with iPS cells generated from autologous skin. Science. 2007;318(5858): 1920-3. doi:10.1126/science.1152092.

33. Sebastiano V, Maeder ML, Angstman JF, Haddad B, Khayter C, Yeo DT, et al. In situ genetic correction of the sickle cell anemia mutation in human induced pluripotent stem cells using engineered zinc finger nucleases. Stem Cells. 2011;29(11):1717-26. doi:10. 1002/stem.718.

34. Zou J, Mali P, Huang X, Dowey SN, Cheng L. Site-specific gene correction of a point mutation in human iPS cells derived from an adult patient with sickle cell disease. Blood. 2011;118(17):4599608. doi:10.1182/blood-2011-02-335554.

35. Zou J, Sweeney CL, Chou BK, Choi U, Pan J, Wang H, et al. Oxidase-deficient neutrophils from X-linked chronic granulomatous disease iPS cells: functional correction by zinc finger nuclease-mediated safe harbor targeting. Blood. 2011;117(21): 5561-72. doi:10.1182/blood-2010-12-328161.

36. Chang CJ, Bouhassira EE. Zinc-finger nuclease-mediated correction of alpha-thalassemia in iPS cells. Blood. 2012;120(19):390614. doi:10.1182/blood-2012-03-420703.

37.• Bauer DE, Kamran SC, Lessard S, Xu J, Fujiwara Y, Lin C, et al. An erythroid enhancer of BCL11A subject to genetic variation determines fetal hemoglobin level. Science. 2013;342(6155):253-7. doi:10.1126/science.1242088. The use of engineered nucleases to validate a regulatory element that is now a potential therapeutic target for genome editing. 